\title{
Editorial
}

\section{Korea Prepares to Achieve the Smart Society}

The Korean Government is rapidly moving from recognition that Information Technology is "the key driver that brings social and economic megatrends and at the same time fundamentally changes the national system." It is now applying IT to cover a broad range of national and social issues including large scale disasters with the goal of achieving the Smart Society.

The National Information Society Agency (NIA) under the leadership of President Dr. Seang Tae Kim, is the designer of smart strategies that cover the entire nation. First steps involve the "smart e-government service infrastructure." Dr. Kim outlines the changes in the e-Government paradigm required to achieve the Smart Society. NIA has provided an extensive description of
Korea's experience and future directions for achieving its Smart Society presented in a Special Report in this issue of the Journal.

While the focus of the United Nations is on eGovernment rankings to measure progress in introducing technical and administrative reforms, Korea with its Smart Society initiatives is clearly leading the world in adopting "transformative" initiatives that are only speculated about by most national governments and international organizations.

Russell Pipe, Editor in Chief 